\title{
Caracterización fisicoquímica de los aceites crudos de algunas semillas de leguminosas del desierto sonorense
}

\author{
Por M. Ortega-Nieblas (1) y L. Vázquez-Moreno (2) \\ (1) Centro de Investigaciones Científicas y Tecnológicas de la Universidad de Sonora. \\ Apdo. Postal 1819. Hermosillo. Sonora. 83000. México. \\ (2) Centro de Investigación en Alimentación y Desarrollo. A. C. Apdo. Postal 1735. Hermosillo. Sonora. 83000. México
}

RESUMEN

Caracterización fisicoquímica de los aceites crudos de algunas semillas de leguminosas del desierto sonorense.

El desierto de Sonora cuenta con una gran variedad de plantas silvestres principalmente de la familia leguminosae de las cuales se tiene poca información acerca de su potencial como fuente nutricional. Por lo que se estudiaron los aceites de las semillas: Acacia famesiana (huizache), Mimosa grahamii (gatuña), Cercidium microphyllium (palo verde 1), Cercidium sonorae (brea), Parkinsonia aculeata (palo verde 2), Olneya tesota (palo fierro) y Prosopis juliflora (mezquite).

Los aceites de cada semilla se extrajeron con hexano, obteniéndose de 8.5 a $23.5 \%$. Estos mostraron un alto grado de insaturación reflejado en los índices de iodo, de 101 a 147. Los índices de acidez, peróxidos y ácidos grasos libres fueron bajos y dentro de los valores aceptables. Al cuantificar los ácidos grasos por cromatografía de gases, predominaron los ácidos linoleico y oleico. De acuerdo a los parámetros analizados todos los aceites crudos resultaron de buena calidad, comparable a la de los aceites de soja, maíz, girasol y cártamo.

PALABRAS-CLAVE: Aceite de semilla - Desierto de Sonora - Estudio físico-químico - Leguminosa - Méjico.

\section{SUMMARY}

Physicochemical characterization of raw oils from some Sonoran desert leguminous seeds.

The Sonoran desert has a great variety of nutritional native plants, mainly from the leguminous family. Although in ancient times their products were used as food by native Americans, there is little information about their potential as a food source. For this reason, oils from the following seeds were studied: Acacia famesiana (huizache), Mimosa grahamii (gatuña), Cercidium microphyllium (palo verde 1), Cercidium sonorae (brea), Parkinsonia aculeata (palo verde 2), Olneya tesota (palo fierro) and Prosopis juliflora (mezquite).

Oils were extracted from the seeds with hexane, and contents ranged from 8.5 to $23.5 \%$. The physicochemical studies showed mostly unsaturated oils, as demonstrated by the iodine index, (101-147 units). Indeces of acidity, peroxides and free fatty acids were low and within the accepted values. Fatty acids were separated and quantified by gas chromatography. Linoleic and oleic acids were found to predominate. All extracted crude oils were of good quality, comparable to those from soybean, com, sunflower and carthamus.

KEY-WORDS: Leguminous - Mexico - Physico-chemical study - Seed oil - Sonoran desert.

\section{INTRODUCCION}

Tradicionalmente se considera que las leguminosas son buenas fuentes de proteína y algunas también tie- nen altos contenidos de aceite (4-6). En muchos países las proteínas y los aceites de origen vegetal son los únicos o los mayores aportadores de energéticos (7). La deficiencia de grasas en muchas ocasiones es provocada por el suministro inadecuado de aceites vegetales $(5,6)$. El problema puede ser de gran importancia si se sabe que de los 36 mejores cultivos de oleaginosas sólo siete (maíz, algodón, olivo, ajonjolí, girasol, cártamo y soja) pueden ser cultivadas bajo condiciones de relativa sequedad $(8,3,4,10,16)$.

El desierto sonorense en su gran extensión $(310,000$ $\left.\mathrm{Km}^{2}\right)$, cuenta con aproximadamente 375 especies nativas y 75 adaptadas o naturalizadas $(4,8)$. Por sus características agronómicas el $10 \%$ de ellas se pueden utilizar para mejorar los recursos de alimentación de la región y del país (8). Dentro de estas leguminosas, los géneros de Prosopis, Acacia, Cercidium, Parkinsonia, Olneya y Mimosa sobresalen por su abundancia y características agronómicas, no necesitan de una gran cantidad de agua, energía o minerales para tener una máxima productividad $(8,9)$. Aunado a esto, está la capacidad de fijar nitrógeno $(9,15)$, que las hace atractivas a ser domesticadas $(8,9)$. Dado su potencial agronómico se juzgó de interés, caracterizar fisicoquímicamente los aceites provenientes de las semillas de brea, palo verde 1, palo fierro, huizache, palo verde 2 , gatuña y mezquite.

\section{DISTRIBUCION Y DESCRIPCION BOTANICA DE LAS SEMILLAS SILVESTRES ESTUDIADAS}

Los géneros empleados para el presente estudio fueron: Parkinsonia, Acacia, Cercidium, Prosopis, Mimosa y Olneya. Las cuales se describen a continuación y su distribución en los Estados de Sonora, Baja California y Baja California Sur se aprecian en la Figura 1.

\section{Cercidium microphyllium (palo verde 1).}

Planta de la familia leguminosae. Se localiza en gran abundancia en México en los Estados de Sonora y Baja California, y en los Estados Unidos de Norte América, en Colorado y California, así como en Venezuela y Perú (13). 


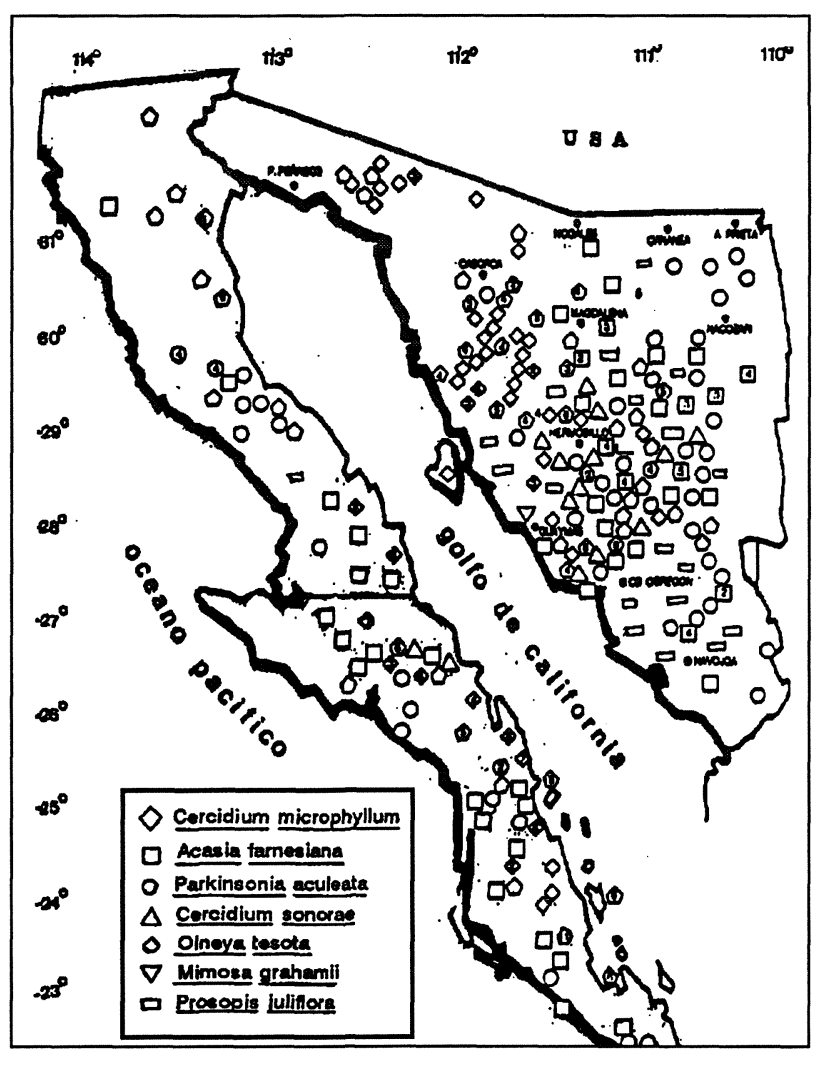

Figura 1

Distribución de las especies en los estados de Sonora, Baja California y Baja California Sur.

Es un árbol de $8 \mathrm{~m}$ de alto, con ramas delgadas de color verde amarillento, tiene de 4-6 pares de hojillas anchas y elípticas de 1-2 $\mathrm{mm}$ de largo. Su período de floración y producción del fruto es en los meses de marzo a mayo. Las vainas son moniliformes de $4-8 \mathrm{~cm}$ de largo de color café, las semillas son ovoides de color verde, elípticas y tienen una longitud de $8-10 \mathrm{~mm}$.

\section{Parkinsonia aculeata (palo verde 2).}

Leguminosa localizada en México en gran abundancia. Se localiza alrededor de arroyos y en tierras arenosas, en zonas bajas del Estado de Sonora y zonas tropicales al sur de México (13). Este árbol llega a medir $12 \mathrm{~m}$ de altura, consta de ramas delgadas hacia abajo, se protege con espinas de 0.4 a $3 \mathrm{~cm}$ de largo, sus tallos son de color verde y sus hojas son cortas y pecioladas. Las vainas son lineales y cilíndricas de 3 a $18 \mathrm{~cm}$ de largo las semillas son estrechas longitudinalmente, son ovoides de 4-5 mm de diámetro, de 8-10 $\mathrm{mm}$ de largo, son lisas y brillantes de color verde combinadas con manchas café.

\section{Cercidium sonorae (brea).}

Planta localizada en suelos áridos y en pendientes en la zona baja de Sonora, en la cercanía de Magdalena Sonora, y en lugares planos al sur de Guaymas Sonora. Es un árbol de madera fina de 6 a $8 \mathrm{~m}$ de alto, con ramas puberulentas y protegidas con espinas rectas de 5 a $15 \mathrm{~mm}$ de largo; con 4 a 6 pares de hojas redondeadas oblongadas de 1.5 a $2 \mathrm{~mm}$ de ancho, el tallo es de color verde brillante y encerados. Las vainas son comprimidas de 7 a 8 $\mathrm{mm}$ de ancho y de 4 a $7 \mathrm{~cm}$ de largo, con estrechamiento de semillas, por lo general contiene de 1 a 4 semillas lenticuladas oblongadas (14).

\section{Olneya tesota (palo fierro).}

Es muy abundante en el desierto de Sonora, al sur y suroeste de Sonora. Arbol de $8 \mathrm{~m}$ de altura, su corteza es dura y brillante, sus hojas son delgadas y verdes que pierde en tiempo de sequía o fríos muy intensos. El fruto es una vaina pequeña indiscente, que contiene de dos a tres semillas, las cuales son de color negrusco, ovaladas de 8 $\mathrm{mm}$ de largo, con apariencia de enceradas. La madera externa es de color amarillo claro con un espesor de 2 a 4 $\mathrm{cm}$, la madera del centro es café oscuro y es extremadamente dura (13).

\section{Acacia farnesiana (huizache).}

Planta localizada en México en las zonas bajas del Estado de Sonora, Hidalgo, San Luis Potosí, Nuevo León, Tamaulipas y en Baja California, así como en otras partes del continente Americano (14). El árbol llega a medir $9 \mathrm{~m}$ de alto, con ramas puberulentas de color gris, espinas rectas estipuladas de 0.5 a $5 \mathrm{~cm}$ de largo, de 8 a 25 pares de hojillas de forma lineal a oblongadas y de 2 a $6 \mathrm{~mm}$ de largo. Las vainas son redondas y turgentes de 4 a $7 \mathrm{~cm}$ de largo y $1.5 \mathrm{~cm}$ de diámetro, son finamente venoreticuladas y su semilla es de color negro de 8 a $10 \mathrm{~mm}$ de largo.

\section{Mimosa grahamii (gatuña).}

Se encuentra en las pendientes rocosas y cañadas, pero sobre todo en las partes más altas de la región de Sonora, en el Estado de Arizona y al sureste de Nuevo México (13). Es un árbol de $7 \mathrm{~m}$ de altura, con espinas rígidas y cortas de 6 a 8 pares. Las hojas son oblongadas de ápice agudo o redondeado de 4 a $6 \mathrm{~mm}$ de largo; las vainas son oblongadas y redondeadas hasta el ápice de 7 a 9 $\mathrm{mm}$ de ancho y de 3 a $4.5 \mathrm{~cm}$ de largo. Las semillas son de color gris, ovoides y planas de 5 a $7 \mathrm{~mm}$ de ancho y de 3 a $7 \mathrm{~cm}$ de largo, con centro negrusco.

\section{Prosopis juliflora (mezquite).}

Leguminosa que se encuentra distribuida en gran abundancia en el desierto de los Estados de Baja California, Chihuahua y Sonora, y al sur de los Estados Unidos de Norte América (14). Es un árbol de 8 a $12 \mathrm{~m}$ de altura, adaptado a suelos áridos y resistentes a sequía, como todas las especies anteriores. Tiene hojas bipinnadas, su corteza es dura, su fruto es una vaina que mide alrededor de $10 \mathrm{~cm}$ de largo y de $8 \mathrm{~mm}$ de ancho de color café oscuro, sus semillas son compactas de 8 a $10 \mathrm{~mm}$ de largo oblongadas (12). 


\section{PARTE EXPERIMENTAL}

La recolección de las vainas se realizó de una misma planta y en el mismo lugar por especie. Se muestreó de diferentes partes de una misma planta (superior, inferior y alrededor de ella) y las vainas se colocaron en bolsas de plástico. La gatuña y brea se cosecharon en el municipio de Cajeme, Sonora, en la carretera Esperanza-Tezopaco. Las especies de palo verde 1, palo verde 2 y palo fierro se recolectaron al sur del municipio de Hermosillo, Sonora y las especies de huizache y mezquite se obtuvieron al suroeste de Hermosillo.

Cada una de las harinas correspondientes a las semillas, fueron extraídas con hexano, durante $8 \mathrm{~h}$., de acuerdo con el método intermitente Soxhlet, según la técnica No 920.39 de (2). De la micela obtenida se separó el aceite a presión reducida y a $55^{\circ} \mathrm{C}$, mediante un rotavapor BauschLomb. El aceite fue secado en estufa a $37^{\circ} \mathrm{C}$ durante $2 \mathrm{~h}$. para eliminar totalmente el hexano residual y almacenado a $4^{\circ} \mathrm{C}$ hasta su uso. Todos los análisis fueron realizados por duplicado o triplicado de acuerdo con la variabilidad de la técnica.

\subsection{Análisis físicos y químicos}

Fueron llevados a cabo de acuerdo con los métodos oficiales recomendados por A.O.C.S. (1).

- Punto de fusión: Método Cc 1-25 (1).

- Indice de refracción: Método Cc 7-25 (1), utilizando un refractómetro de Abbe Bausch \& Lomb, modelo 31.

- Indice de lodo: Método de Hanus. A.O.A.C. 28.018 (2).

- Indice de Saponificación: Método Cd 3-25 (1).

- Indice de Acidez: Método Cd 3a-63 (1).

- Indice de Peróxido: Método Cd 8-53 (1).

- Acidos Grasos Libres: Método Ca 5-40 (1).

\subsection{Análisis cromatográficos}

Determinación de ácidos grasos. Los triglicéridos se saponificaron y los ácidos grasos se metilaron, utilizando hidróxido de sodio y metanol, de acuerdo con el método $\mathrm{Ce}-27$ (1).

Cuantificación de los ácidos grasos. Se efectuó por cromatografía de gases (cromatógrafo Perkin Elmer, 8410), utilizando un detector de ionización de llama, con una sensibilidad de $2 \times 10-10$ AFS. Una columna de acero inoxidable de 4 pies de largo por 0.5 plg de diámetro con empaque DEGS $10 \%$ sobre un soporte de $80 / 100$ chromosorb W-HP. El nitrógeno se usó como gas portador a una velocidad de flujo de $15 \mathrm{ml} / \mathrm{min}$. La temperatura del detector y del inyector fue de $200^{\circ} \mathrm{C}$. Los datos fueron procesados en un integrador del cromatógrafo. Todos los aceites se analizaron por duplicado.

\section{RESULTADOS Y DISCUSION}

Los aceites de las especies silvestres analizadas se extrajeron con una eficiencia del 90 al $100 \%$. Todos los aceites crudos presentaron un color amarillo transparente, sin olores desagradables y fueron analizados sin necesidad de ser refinados o neutralizados. En la Tabla I se presenta el porcentaje de aceite encontrado en cada una de las semillas. La concentración más alta correspondió al de gatuña, siguiéndole palo verde 1, brea y mezquite. Los contenidos más bajos se presentaron en las semillas de huizache, palo verde 2 y palo fierro. Al ser comparados con fuentes tradicionales comerciales, la semilla de palo verde 1 tuvo un porcentaje igual al reportado para la soja (18.7\%); el porcentaje de aceite de gatuña $(23.5 \%)$ es cercano al del ajonjolí (27\%). Además, esta semilla tiene más aceite que la de soja y la de maíz (4.5\%). En general, se puede considerar que los porcentajes de aceites en las especies estudiadas son mayores o comparables a las fuentes de uso tradicional $(3,5)$.

Tabla I

Porcentaje de aceite en las semillas de las plantas estudiadas

\begin{tabular}{lc}
\hline \multicolumn{1}{c}{ Semillas } & \% Aceite \\
\hline C. sonorae (brea) & 16.5 \\
C. microphyllium (palo verde 1) & 18.2 \\
P. aculeata (palo verde 2) & 8.5 \\
O. tesota (palo fierro) & 10.3 \\
M. grahamii (gatuña) & 23.5 \\
A. farnesiana (huizache) & 12.0 \\
$P$. juliflora (mezquite) & 14.5 \\
\hline
\end{tabular}

a: Extracción de $100 \mathrm{~g}$ de semilla.

La mayoría de los aceites estudiados presentaron un alto grado de insaturación ( 86 a $87 \%$ ). Los porcentajes de ácidos grasos son similares a los reportados para soja y maíz, y ligeramente menores que el de girasol (3). Los contenidos de ácidos grasos saturados (14\%) con excepción de palo verde 1 , fueron muy parecidos a los comerciales (3).

Como se esperaba el alto grado de insaturación de los aceites se vió reflejado en los índices de iodo (I.I), (Tabla II), éstos variaron de 101 a $147 \mathrm{mg}$ de $\mathrm{KI} / \mathrm{g}$. A excepción del aceite de palo fierro, todas las demás especies tienen I.I. superiores a los reportados para el de maíz, colza, soja y uña de gato (3, 5 y 11). Los índices de acidez (I.A.), peróxidos (I.P.) y el contenido de ácidos grasos libres (A.G.L.), fueron bajos. Los aceites de colza y soja, cuyos índices de acidez son relativamente altos, deben ser refinados para reducirlos $(3,5)$. Los valores de los puntos de fusión (P.F.) y de los índices de refracción (I.R.) fueron similares entre sí y a los de los aceites de fuentes comparativas, mostrándose en la Tabla II. Todos los aceites estudiados están dentro de las normas establecidas por la Asociación Americana de Química de los Aceites, AOCS (1). 
Tabla II

Comparación de los parámetros físico-químicos de los aceites de especies silvestres y comestibles

\begin{tabular}{|c|c|c|c|c|c|c|c|}
\hline Semillas & $\begin{array}{l}\text { P.F. } \\
{ }^{\circ} \mathrm{C}\end{array}$ & $\begin{array}{l}\text { I.R. } \\
25^{\circ} \mathrm{C}\end{array}$ & $\begin{array}{c}\mathrm{I.I.} \\
\mathrm{mg} \mathrm{KI} / \mathrm{g}\end{array}$ & $\begin{array}{c}\text { I.A. } \\
\mathrm{mg} \mathrm{KOH/g}\end{array}$ & $\begin{array}{c}\text { I.S. } \\
\text { mgNaOH }\end{array}$ & $\begin{array}{c}\text { I.P. } \\
\text { meq/g }\end{array}$ & $\begin{array}{c}\text { A.G.L. } \\
\%\end{array}$ \\
\hline Brea & $13-10$ & 1.47 & 131 & 0.70 & 81 & 3.7 & 0.70 \\
\hline P. verde 1 & $10-8$ & 1.48 & 136 & 0.56 & 79 & 3.6 & 0.51 \\
\hline P. fierro & $13-8$ & 1.47 & 101 & 0.52 & 78 & 5.6 & 0.60 \\
\hline Gatuña & $13-10$ & 1.45 & 147 & 0.42 & 100 & 4.0 & 0.32 \\
\hline P. verde 2 & $10-8$ & 1.47 & 134 & 0.60 & 82 & 3.8 & 0.70 \\
\hline Huizache & $13-10$ & 1.48 & 136 & 0.58 & 80 & 3.5 & 0.61 \\
\hline Mezquite & $10-7$ & 1.45 & 130 & 0.63 & 76 & 2.8 & 0.72 \\
\hline Soja $^{a}$ & $13-10$ & 1.47 & 130 & 1.2 & 192 & 7.3 & 0.50 \\
\hline Girasola $^{a}$ & $10-9$ & 1.47 & 130 & 0.67 & 191 & 6.2 & 0.63 \\
\hline Maíz & $11-8$ & 1.47 & 115 & 0.51 & 98 & 3.5 & 1.30 \\
\hline Colza $^{\mathrm{b}}$ & $10-7$ & 1.47 & 108 & 1.00 & 170 & 3.2 & 0.56 \\
\hline Uña de gato ${ }^{\circ}$ & $13-9$ & 1.47 & 110 & 0.56 & 157 & 5.3 & 0.61 \\
\hline
\end{tabular}

a: Baileys, 1979; b: Bernandini, 1980; c: Ortega y Vázquez-Moreno, 1993.

La composición de los ácidos grasos de los aceites se presenta en la Tabla III. Dentro de los ácidos grasos insaturados predominó el linoleico, con excepción de la semilla de palo verde 1, que tiene mayor contenido de oleico. Entre los ácidos grasos saturados predominó el palmítico.

Tabla III

Comparación de la composición de los acidos grasos de los aceites de semillas silvestres y comestibles

\begin{tabular}{|c|c|c|c|c|c|c|c|c|c|}
\hline \multirow[t]{2}{*}{ Semillas } & $\mathbf{C}_{16}$ & $\mathbf{C}_{16: 1}$ & $C_{18}$ & $\mathbf{C}_{18: 1}$ & $C_{18: 2}$ & $C_{20}$ & $C_{18: 3}$ & $C_{20: 4}$ & $\mathbf{C}_{22: 1}$ \\
\hline & \multicolumn{9}{|c|}{ (n) Porcentajes } \\
\hline Brea & 8.4 & 0.24 & 4.2 & 36 & 47 & 1.0 & 0.5 & 1.5 & 0.4 \\
\hline P. verde 1 & 12.6 & 0.70 & 9.0 & 51 & 21 & 1.8 & 0.6 & 1.5 & 0.7 \\
\hline P. verde 2 & 7.7 & 0.20 & 4.7 & 35 & 47 & 1.7 & 0.6 & 1.6 & 0.3 \\
\hline Palo fierro & 8.3 & 0.22 & 4.6 & 35 & 48 & 1.0 & 0.5 & 0.7 & 0.2 \\
\hline Gatuña & 8.0 & 0.22 & 3.9 & 35 & 50 & 1.0 & 0.6 & 0.9 & 0.2 \\
\hline Huizache & 7.5 & 0.20 & 5.0 & 18 & 67 & 0.4 & 0.8 & 0.3 & 0.0 \\
\hline Mezquite & 7.8 & 0.20 & 4.1 & 35 & 50 & 1.0 & 0.6 & 0.7 & 0.2 \\
\hline Soja $a^{a}$ & 11.0 & 0.52 & 4.5 & 28 & 50 & 1.0 & 8.1 & 0.5 & 1.5 \\
\hline Girasola $^{a}$ & 3.6 & 0.30 & 3.0 & 34 & 57 & 1.2 & 0.8 & 1.1 & 2.1 \\
\hline Maíz $z^{\mathrm{a}}$ & 7.8 & 0.20 & 3.6 & 46 & 41 & 0.4 & 0.8 & 0.9 & 1.3 \\
\hline Uña de gatob & 8.1 & 0.40 & 3.7 & 35 & 52 & 0.2 & 0.4 & 0.2 & 1.1 \\
\hline
\end{tabular}

a: Baileys, 1979; b: Ortega-Nieblas M. y Vázquez-Moreno, L. 1993. 
La concentración del ácido oleico varió de 18 a 51\%, correspondiendo al aceite de huizache y al de palo verde 1 respectivamente, Tabla III. En general el porcentaje de este ácido graso en los aceites de plantas silvestres fue mayor que el reportado para soja y girasol. Mas aún, palo verde 1 está sobre cualquiera de las especies comerciales. Algunas semillas tuvieron proporciones de ácido linoleico muy altas, el mayor porcentaje correspondió al aceite de huizache $(67 \%)$ que a su vez es similar al reportado para cártamo (5). A excepción de la semilla de palo verde 1 , todas las demás tienen contenidos de ácido linoleico igual o ligeramente menor a los comerciales.

\section{CONCLUSIONES}

Los aceites de las especies silvestres estudiadas presentaron características químicas y fisicoquímicas iguales o superiores a los usados de manera tradicional. Por sus características se considera que podrían competir o complementarse con los aceites de maíz, girasol, cártamo, ajonjolí y colza que son producidos a mayores costos y en condiciones climatológicas y agronómicas especiales. Los aceites estudiados son estables, no manifestaron rancidez o inicio de oxidación al ser almacenados por corto tiempo y tampoco requirieron de refinación o procesado. No se debe descartar la posibilidad de su utilización en la industria alimentaria (margarinas y mayonesas) y de pinturas.

\section{BIBLIOGRAFIA}

1. AOCS. American Oil Chemist Society. (1989).- Official and Recommended Practices of the Amer. Oil Chem. Soc. Champaign, IL.

2. AOAC. (1990).- Association of Official Analytical Chemists, Official Methods of Analysis.- (15th edn).- Washington, DC, USA.
3. Baileys, A. E. (1979).- Aceites y Grasas Industriales.- Ed. Reverté, Argentina. S.C.A. Vol.II, Pags. 432-440.

4. Benson, L. (1979).- "Trees and Shrubs of the Southwestern Desert".3ra. ed.- The University of Arizona Press, Tucson, Arizona. Pag. 416.

5. Bernandini, E. y Franco, J. (1981).- "Tecnología de aceites y grasas".1ra. ed.- Editorial Alhambra. Madrid, España.

6. Christie, W. (1972).- "Topics in Lipid Chemistry".- Vol. III. ed. by Logos Press, London, England, Pag. 171-183.

7. Elías, L. y Bresani, R. (1978).- "Composición Química y Valor Nutritivo de Algunas Leguminosas de Grano".- Turrialba. INCAP. 26 (4), 375.

8. Felger, R., and Nabhan, G. (1978).- "Agroecosystem: A Model from the Sonoran Desert". In "Social and Technological Management in Dry Lands. Past and Present, In degenous and imposed".- ed. AAAS, Selected Symposium, No. 10, pag. 129-148.

9. Hernández, M. y Bouges, H. (1983).- "Valor Nutritivo de los Alimentos Mexicanos".- Publicación L-12 de la División de Nutrición. Instituto Nacional de Nutrición. Salvador Zubiran, México, D.F.

10. Nabhan, G. y Weber, C. (1985).- "Variation in Composition of Hopi Indian Beans".- Ecology of Food and Nutrition 16, 135-145.

11. Ortega-Nieblas, M. y Vázquez-Moreno, L. (1993).- "Caracterización Fisicoquímica del Aceite Crudo y Refinado de la Semilla de Proboscidea parviflora (uña de gato)".- Grasas y Aceites 44, 30-34.

12. Signoret, P. (1970).- "Datos Sobre Algunas Características del Mezquite y su Aprovechamiento en el Valle del Mezquital". En "Mezquites (huizaches)".- IMRNR México. Pag. 73-123.

13. Shreve, F. y Wiggins, I. (1986a).- "Vegetation and Flora of the Sonora Desert".- Stanford University Press, USA, Vol. I. Pags. 143-176.

14. Shreve, F. y Wiggins, I. (1986b).- "Vegetation and Flora of the Sonora Desert".- Stanford University Press, USA, Vol. II. Pags. 1231-1265.

15. Sotelo, A. (1981).- "Leguminosas Silvestres, Reserva de Proteínas para la Alimentación del Futuro".- Información Científica y Tecnológica 3, 54-58

16. Swern, A. (1964).- "Food Oils and Their Uses".- Avi, Publishing Company. Inc. USA. New York.

Recibido: Mayo 1994

Aceptado: Febrero 1995 